\title{
Evaluación de la prescripción de glibenclamida en diabéticos tipo 2
}

\section{Evaluation of glibenclamide prescription in type 2 diabetic patients}

\author{
Rodolfo Llinás Castro ${ }^{1}$, Luis Alvis Estrada², Luis Mendoza Goez ${ }^{2}$
}

Forma de citar: Llinas Castro R, Alvis Estrada L, Mendoza Goez L. Evaluación de la prescripción de glibenclamida en diabéticos tipo 2. Rev Univ Ind Santander Salud. 2017; 49(1): 9-15. DOI: http://dx.doi.org/10.18273/revsal.v49n1-2017001 @) (1)

\section{RESUMEN}

Introducción: Las sulfonilureas permanecen junto con la metformina como el tratamiento farmacológico más usado para los pacientes diabéticos tipo 2 , y continúan siendo los antidiabéticos más prescritos en algunas regiones del mundo. Objetivo: Evaluar la prescripción de glibenclamida en aspectos relacionados a contraindicaciones, dosificación, y prevención de efectos adversos, en el tratamiento de los pacientes diabéticos tipo 2 que acuden a una institución de salud de baja complejidad en Cartagena de Indias, Colombia. Metodología: Estudio descriptivo con enfoque desde la fármaco-epidemiología dirigido específicamente al campo de los estudios de utilización de medicamentos, basado en los datos recogidos mediante revisión de historias clínicas de 331 pacientes diabéticos tipo 2, mayores de 18 años en un centro de atención primaria en salud de la ciudad de Cartagena de Indias entre los años 2013 y 2014. Se estudiaron variables demográficas, clínicas y paraclínicas, el esquema terapéutico y la calidad de la prescripción. La información se analizó usando estadística descriptiva. Resultados: 57 \% (189) de los pacientes utilizaron glibenclamida siendo un $59.3 \%$ (153) prescrito de manera inadecuada, $45.5 \%$ (86) tenían alguna contraindicación para el empleo del medicamento. En $1 \%$ de los pacientes de la muestra se prescribió sobrepasando el rango de dosis terapéutica Conclusiones: La calidad de la prescripción de la glibenclamida fue inadecuada en una proporción importante de pacientes, se hace necesario implementar estrategias educacionales que capaciten al personal médico para una correcta utilización de los fármacos antidiabéticos. La glibenclamida comparada con otras sulfonilureas de segunda generación nos brinda más desventajas que ventajas.

Palabras Clave: Diabetes Mellitus Tipo 2, glibenclamida, contraindicaciones, tasa de filtración glomerular, prescripciones de medicamentos, farmacoepidemiología.

\footnotetext{
ABSTRACT

Introduction: Sulfonylureas remain with metformin as the most used drug treatment for type 2 diabetic patients and remain the most prescribed anti-diabetic in some regions of the world. Target: Evaluate prescribing glyburide in aspects related to contraindications, dosage, and prevention of adverse effects, in the treatment of type 2 diabetic patients attending a health institution low complexity in Cartagena de Indias, Colombia. Methodology: Descriptive study with approach from the pharmaco-epidemiology specifically directed to the field of studies of drug use, based on the data collected through review of medical records of 331 type 2 diabetic patients over 18 years in a primary

1. Empresa Social del Estado ESE Hospital Local Cartagena de Indias. Bolivar, Colombia

2. Universidad de Cartagena, Bolivar. Colombia

Correspondencia: Rodolfo Llinás Castro. Dirección: Pie de la Popa, Calle 33 22-54. Corrreo electónico: rodolfollinas@yahoo.com. Teléfono: (5) 6505898 . 
health care in the city of Cartagena de Indias between 2013 and 2014. demographic, clinical and paraclinical variables, the therapeutic plan and prescription quality were studied. Data was analyzed using descriptive statistics. Results: $57 \%$ (189) patients using glyburide. In 59.3\% (153) inappropriately prescribed glibenclamide. 45.5\% (86) of the patients had a contraindication to the use of the drug. In $1 \%$ of patients were prescribed the sample exceeding the therapeutic dose range Conclusions: The quality of prescribing glyburide was inadequate in a substantial proportion of patients, it is necessary to implement educational strategies that enable medical personnel to ensure proper use of antidiabetic drugs. Glyburide compared with other second-generation sulfonylureas gives us more disadvantages than advantages.

Keywords: Diabetes Mellitus, Type 2, Glyburide, /contraindications, Glomerular Filtration Rate, drug prescriptions, pharmacoepidemiology.

\section{INTRODUCCIÓN}

En la actualidad existe un número muy variado de fármacos antidiabéticos con diferentes mecanismos de acción ${ }^{1}$. A pesar de ello las sulfonilureas se constituyen en un grupo de medicamentos ampliamente utilizados, aun con la introducción de medicamentos como los inhibidores de dipeptidil- peptidasa-IV y los análogos del péptido similar al glucagón tipo $1^{2}$. Estos continúan siendo los antidiabéticos más prescritos en algunas regiones del mundo $^{3}$, permaneciendo junto con la metformina como el tratamiento farmacológico más usado ${ }^{4}$.

El patrón de prescripción de las sulfonilureas dentro del arsenal terapéutico antidiabético se reporta con porcentajes variables: 26,7 y $56 \%$ en Estados Unidos, $40 \%$ en Argentina, 58,2 y 64,9\% en Colombia, subrayando que la sulfonilurea evaluada en Argentina y Colombia fue exclusivamente la glibenclamida ${ }^{5-9}$.

En Colombia, el listado de Medicamentos del Plan de Beneficios en Salud con los que se lleva a cabo el programa de control de la diabetes incluye únicamente las opciones metformina, glibenclamida e insulinas (humanas y análogas) ${ }^{10}$. La glibenclamida y otras sulfonilureas aparecen en los Listados de Medicamentos Esenciales de diferentes países, e incluso en el publicado por la Organización Mundial de la Salud (OMS) en noviembre 2015 por lo que el conocimiento de su adecuado manejo farmacológico continúa siendo indispensable para lograr un uso racional y así asegurar su seguridad, tolerabilidad y eficacia.

La farmacoterapia ocurre de forma adecuada cuando el usuario utiliza los medicamentos conforme a necesidades de salud correctamente identificadas y, al mismo tiempo, no se generan o agravan por el uso de medicamentos. Fallos en esas condiciones esenciales llevan al sufrimiento humano, incapacidad, reducción de la calidad de vida y muerte.
Por todo lo anterior, este estudio evaluó la prescripción de glibenclamida en aspectos relacionados con dosificación, contraindicaciones, y prevención de efectos adversos en el tratamiento de los pacientes diabéticos tipo 2 que acuden a una institución de salud de baja complejidad en Cartagena de Indias, Colombia.

\section{METODOLOGÍA}

Estudio fármaco-epidemiológico de tipo descriptivo basado en los datos recogidos mediante revisión de historias clínicas de los pacientes diabéticos tipo 2, mayores de 18 años que fueron inscritos al programa de atención de diabetes durante los años 2013 y 2014, y que tuvieron mínimo seis meses de control en una institución de baja complejidad. La población la costituyeron 1340 pacientes inscritos entre los años 2013 y 2014.

Con una prevalencia esperada asumida del $50 \%$, un error de $5 \%$ y un nivel de confianza del $95 \%$, la muestra obtenida fue de 384 historias clínicas. Dado que se trata de una población finita el tamaño de muestra fue ajustado dando como resultado 299 historias clínicas. $\mathrm{Al}$ adicionar un $10 \%$ en caso de pérdidas la muestra definitiva fue de 331. Para la selección de la muestra se utilizó un muestreo aleatorio simple del listado de pacientes utilizando la herramienta Muestra de Microsoft Excel ${ }^{\mathbb{}}$.

Los datos del estudio que se recolectaron entre agosto y septiembre de 2015 provenientes de las historias clínicas fueron: sexo, edad, índice de masa corporal (IMC), creatinina sérica, estimación o no de la tasa de filtración glomerular (TFG) por el médico que atiende, resultado de la estimación de la tasa de filtración glomerular calculada por los investigadores, fármaco antidiabético prescrito, presencia de contraindicación para el uso de glibenclamida, dosis prescrita de glibenclamida, prescripción de glibenclamida previa a la ingesta de los alimentos principales. 
Evaluación de la prescripción de glibenclamida en diabéticos tipo 2

En los pacientes que tenían registro de creatinina los investigadores estimaron la tasa de filtración glomerular a través de la ecuación de predicción de CockcroftGault corregida debido a que es la fórmula utilizada en esta institución ${ }^{11}$.

Se consideraron contraindicación para la terapia con glibenclamida: edad mayor a 60 años, enfermedad renal con tasa de filtración glomerular $<60 \mathrm{ml} / \mathrm{min} / 1.73$ $\mathrm{m}^{2}$ de superficie corporal, enfermedad hepática grave (Grado C de la Escala de Child-Pugh) ${ }^{12-17}$. Todos aquellos pacientes sin creatinina disponible en sus registros fueron considerados con una contraindicación para el uso de glibenclamida.

Como rango de dosis terapéutica se definió 2.5 a 20 mg de glibenclamida y se determinó que se había realizado prevención del principal efecto adverso, el cual es la hipoglucemia, cuando en la historia clínica se encontraban registrado que el medicamento se prescribe antes de la ingesta de alimentos.

Se definió prescripción adecuada de glibenclamida cuando se reúnen las tres siguientes condiciones: 1) no exista contraindicación por alguna condición del paciente, 2) prescripción de la dosis dentro del rango terapéutico y 3) prevención de aparición de efecto adverso. La ausencia de una de estas tres condiciones determina la prescripción como inadecuada.

Los datos fueron almacenados en una hoja de cálculo de Microsoft Excel y se llevó a cabo el análisis mediante el programa estadístico SPSS versión 21.0. Del análisis estadístico se obtuvieron tablas que reportan frecuencias absolutas y relativas así como medidas de tendencia central y de dispersión para las variables cuantitativas.

La investigación se basó en lo estipulado por la Resolución 008430 de 1993 del Ministerio de Salud de Colombia y los aspectos éticos contemplados en la declaración de Helsinki. Este proyecto se clasificó como una investigación sin riesgo por parte de la Empresa Social del Estado Hospital Local Cartagena de Indias.

\section{RESULTADOS}

En la Tabla 1 se muestran las características demográficas, clínicas y paraclínicas de los pacientes. De la población de estudio el 61,3\% (203) son del sexo femenino. La media de edad para el grupo de estudio en general fue de 54,3 años (DE=12,2). El 73,7\% (244) de los diabéticos tipo 2 tenían IMC $\geq 25 \mathrm{~kg} / \mathrm{m}^{2}$ y un porcentaje correspondiente al 1,5\% (5) tenían un IMC $<18,5 \mathrm{Kg} / \mathrm{m}^{2}$.
Tabla 1. Características demográficas, clínicas y paraclínicas de los pacientes diabéticos tipo 2 pertenecientes al estudio. Cartagena de Indias, Colombia.

\begin{tabular}{|c|c|c|}
\hline SEXO & n & $\%$ \\
\hline Femenino & 203 & 61,3 \\
\hline Masculino & 128 & 38,7 \\
\hline \multicolumn{3}{|c|}{ CATEGORIA IMC } \\
\hline Bajo peso & 5 & 1,5 \\
\hline Normo peso & 82 & 24,8 \\
\hline Obesidad & 117 & 35,3 \\
\hline Sobrepeso & 127 & 38,4 \\
\hline \multicolumn{3}{|c|}{ CREATININA } \\
\hline No & 35 & 10,6 \\
\hline $\mathrm{Si}$ & 296 & 89,4 \\
\hline \multicolumn{3}{|c|}{ CALCULO TFG } \\
\hline No & 144 & 43,5 \\
\hline $\mathrm{Si}$ & 187 & 56,5 \\
\hline Total & 331 & 100,0 \\
\hline
\end{tabular}

En el 10,6\% (35) de los pacientes no se encontró registro de creatinina sérica en ninguna de las consultas médicas evaluadas. La tercera parte de los pacientes tuvieron reporte de su creatinina pero a pesar de ello el médico a cargo no estimó la TFG.

El 57\% (189) de los pacientes utilizaron glibenclamida siendo el segundo fármaco más prescrito después de la metformina, la cual fue utilizada por el $84,9 \%$ (281) de los pacientes y la insulina la utilizó el 18\% (61) de los pacientes.

De los pacientes con prescripción de glibenclamida, el $45.5 \%$ (86) contaba con alguna condición referida como contraindicación para el empleo de glibenclamida. Dentro de las condiciones patológicas que son referidas como causa de contraindicación de esta sulfonilurea la causa de mayor motivo de contraindicación fue ser mayor de 60 años (73\%).

Se encontró que una cuarta parte de los pacientes a los que se les prescribió glibenclamida tenían una TFG $<60 \mathrm{ml} / \mathrm{min} / 1,73 \mathrm{~m}^{2}$ de superficie corporal y similar porcentaje de pacientes que utilizaban glibenclamida no contaron con valores de creatinina para estimar la TFG. No se encontró ningún paciente con enfermedad hepática grave.

En la Tabla 2 se muestra el porcentaje de prescripción en rango de dosis, prevención de eventos adversos previstos y de uso inadecuado de glibenclamida. Se 
encontró que dos pacientes correspondientes al $1 \%$ de la muestra sobrepasaban el rango de 2,5 a $20 \mathrm{mg} /$ día de la glibenclamida al prescribirle esta sulfonilurea. En la tercera parte de los pacientes que tienen prescrito este medicamento, no se halló documentado que se les recomienda sea ingerido antes del desayuno, almuerzo o cena.

Sólo 40,7\% (77) de los pacientes que utilizan glibenclamida en este estudio tienen una prescripción adecuada del medicamento según los criterios previamente establecidos.

Tabla 2. Proporción de pacientes con prescripción en rango de dosis, prevención de eventos adversos y de uso inadecuado de glibenclamida en pacientes diabéticos tipo 2. Cartagena de Indias, Colombia.

\begin{tabular}{lcc}
\hline PRESCRIPCION EN RANGO DE DOSIS & $\mathbf{n}$ & $\mathbf{\%}$ \\
\hline $\mathrm{No}$ & 2 & 1,1 \\
$\mathrm{Si}$ & 187 & 98,9 \\
\hline PREVENCIÓN DE EVENTOS ADVERSOS & & \\
\hline No & 66 & 34,9 \\
$\mathrm{Si}$ & 123 & 65,1 \\
\hline
\end{tabular}

\begin{tabular}{|c|c|c|}
\hline \multicolumn{3}{|c|}{ ADECUACION SULFONILUREA } \\
\hline Adecuada & 77 & 40,7 \\
\hline Inadecuada & 112 & 59,3 \\
\hline Total & 189 & 100,0 \\
\hline
\end{tabular}

\section{DISCUSIÓN}

La utilización de cualquier fármaco requiere estricta observación de su farmacología. El desconocimiento o la falta de interés por los conceptos básicos farmacológicos pondrían al paciente frente a un riesgo para su salud.

Estudios como el de Al Khaja evidencian que la prescripción de glibenclamida en mayores de 65 años constituyen un porcentaje muy bajo en errores en la prescripción ${ }^{18}$, contrario a lo hallado en el presente estudio debido a la edad asumida para determinar el error fue desde los 60 años.

En relación a lo anterior, es importante indicar, que aunque en la actualización 2015 los criterios de Beer ${ }^{19}$ señalan como recomendación que la glibenclamida se debe evitar en la población mayor de 65 años debido a más alto riesgo de hipoglucemia severa prolongada, la OMS desde el año 2013 a través de una revisión no sistemática ${ }^{13}$ y otros autores por revisiones sistemáticas ${ }^{12}$, proveen evidencia apoyando la recomendación para su no uso incluso en individuos mayores de 60 años de edad. Aunado a esto en el Listado de Medicamentos Esenciales 2013 y 2015 de la OMS con respecto a la glibenclamida se halla una nota mencionando que esta no es recomendada en pacientes mayores de 60 años $^{20}$.

A pesar que la glibenclamida tiene un $50 \%$ de su excreción por vía renal ${ }^{21}$ y que está contraindicada con una TFG $<60 \mathrm{ml} / \mathrm{min} / 1.73 \mathrm{~m}^{2}$ de superficie corporal ${ }^{14}$, se encontró como uno de los motivos principales el no registro de creatinina sérica y la subsecuente no estimación de la TFG. Esto lo convierte en una limitación del estudio, pero expone la deficiencia en la calidad de la prescripción del medicamento, al no valorarse la función renal para evaluar la restricción de su uso por enfermedad renal, incrementando con ello la posibilidad de presentar una hipoglucemia en aquellos pacientes que pudiesen tener una alteración de su función renal sin ser detectada y en especial si se es mayor a 60 años.

Igualmente, fue una limitante el hecho que en la institución se estime la TFG a través de la ecuación de Cockcroft-Gaut en detrimento de mejores opciones como la MDRD o CKD-EPI, pues los resultados obtenidos con estas últimas correlacionan mejor que el obtenido por Cockcroft-Gault para valores inferiores a $60 \mathrm{ml} /$ $\min / 1,73 \mathrm{~m}^{2}$, que son los mayoritariamente susceptibles de contraindicación de fármacos o necesidad de ajuste de dosis. Incluso las guías recomiendan que el uso de la ecuación de Cockcroft-Gault debiera desaconsejarse ${ }^{22}$.

La proporción de pacientes que sobrepasan el rango de dosis terapéutico máximo resultó igual a la encontrada en Brasil23. Del mismo modo, aunque las sulfonilureas deben ser ingeridas 30 minutos antes de las comidas principales, por varias razones ${ }^{21,24}$ en un tercio de los pacientes no se realizó esta recomendación.

En la literatura escasean estudios sobre el esquema terapéutico de glibenclamida. Aun así, la proporción de prescripciones de glibenclamida adecuadas encontradas en el presente estudio resulta similar al reportado en México, en donde se consideró adecuación a partir de tres factores: número de tabletas, horario de la toma e indicación para tomar el medicamento con o sin alimentos $^{25}$. A pesar de tratarse de aspectos relativamente diferentes, en ambos estudios se observa un bajo porcentaje de adecuadas o correctas prescripciones. Otras publicaciones del mismo país señalan diferentes porcentajes de prescripción adecuada de glibenclamida con valores que oscilan desde 7 hasta $44 \%{ }^{26,27}$. Estos estudios igualmente evalúan diferentes criterios de prescripción adecuada evaluando la indicación, dosificación e intervalo. 
Si los clínicos tienen claras las ventajas, así como las limitaciones de las sulfonilureas estarán en la capacidad de utilizarlas de la manera más eficiente. La monoterapia con sulfonilureas disminuye la $\mathrm{HbA} 1 \mathrm{c}$ alrededor de $1,51 \%$ y se encuentra indicada en población más joven, diabetes de reciente diagnóstico y en pacientes más delgados con $\mathrm{IMC}<27^{16}$, dejando claro que se debe utilizar como segunda o tercera línea terapéutica.

La OMS enlista la gliclazida dentro de las medicinas para el uso en diabetes y registra para la glibenclamida únicamente la advertencia ya mencionada ${ }^{20}$. Este hecho conlleva a lograr un paso importante hacia la seguridad de estos pacientes crónicos con una sulfonilurea mejor tolerada, en oposición a una eficacia a corto término y economía que brindaba la glibenclamida, pero con mayores riesgos.

A pesar de lo anterior, la glibenclamida aún persiste en los Listados de Medicamentos esenciales de la mayoría de los países, ${ }^{28}$ incluido Colombia. Estos Listados son un medidor o calibrador de las prioridades en salud de cada país.

Varios son los motivos para el ingreso de otras sulfonilureas en los listados de medicamentos esenciales en detrimento de la glibenclamida o incluso su reemplazo por otro tipo de medicamentos antidiabéticos de aparición reciente en estos últimos años:

- Mayor riesgo de hipoglucemia que otras sulfonilureas ${ }^{29}$.

- El riesgo de hipoglucemia se incrementa en pacientes con enfermedad renal crónica, en caso de utilizarse una sulfonilurea se recomienda el uso de gliclazida, glipizida, y de glimepirida, está ultima ajustando la dosis ${ }^{14,17}$.

- No recomendada en pacientes mayores de 60 años $^{12}$.

- Diversos estudios muestran que gliclazida y glimepirida tienen un menor aumento de peso, logrando este impacto favorable en los primeros años de uso en comparación con la glibenclamida ${ }^{3}$.

- Gliclazida y glimepirida están asociadas con un más bajo riesgo de mortalidad cardiovascular y mortalidad por todas las causas comparado con glibenclamida $^{30}$

- Glibenclamida a diferencia de las otras dos sulfonilureas mencionadas no preserva el precondicionamiento isquémico miocárdico (mecanismo endógeno de protección cardiaca durante episodios isquémicos), esto ocurre debido a la no selectividad de este fármaco por los receptores SUR1 de las células beta pancreáticas, ligándose también a receptores SUR2A del miocardio y SUR2B de la célula muscular lisa vascular, aun en rangos de dosis terapéuticas ${ }^{30}$.

Por todo lo anterior, la glibenclamida está explícitamente desaconsejada en la mayor parte de las guías y ante esta realidad y teniendo en cuenta la eficacia, seguridad y bajo costo de la gliclazida y glimepirida, la terapia con estas sulfonilureas debe ser considerada como segunda línea después de la metformina en países de bajos ingresos económicos en reemplazo de la glibenclamida ${ }^{31}$.

La reciente disponibilidad de nuevos antidiabéticos como los inhibidores de dipeptidil-peptidasa-IV, los análogos del péptido similar al glucagón tipo $1 \mathrm{y}$ los inhibidores del transportador de sodio-glucosa tipo 2, aunado a estudios que muestran un probable incremento del riesgo de la mortalidad cardiovascular y por todas las causas en los pacientes manejados con sulfonilurea ${ }^{32}$, está llevando a un cambio en las prácticas de prescripción ${ }^{4}$.

\section{CONCLUSIONES}

Al haberse encontrado un alto porcentaje de prescripciones inadecuadas de glibenclamida, se hace necesario implementar estrategias educativas que capaciten al personal médico para una correcta utilización de los fármacos antidiabéticos. En este sentido, las universidades y las sociedades científicas deben jugar un papel primordial, al tiempo que cada institución de salud debería contar con guías de prescripción para los medicamentos utilizados en las principales causas de morbilidad consultadas por sus pacientes, entre ellas la diabetes.

El Ministerio de Salud y Protección Social en Colombia debe revisar a la luz de la literatura científica actual, la permanencia de un medicamento como la glibenclamida dentro del Listado de Medicamentos del Plan de Beneficios en Salud, y, considerar la introducción de otras sulfonilureas que brindan una mayor seguridad y de los nuevos medicamentos antidiabéticos que brindan ventajas sobre las mismas sulfonilureas.

\section{CONFLICTO DE INTERESES}

Los autores declaramos no tener conflicto de intereses. 


\section{REFERENCIAS}

1. Inzucchi SE, Bergenstal RM, Buse JB, Diamant M, Ferrannini E, Nauck M, et al. Management of hyperglycemia in type 2 diabetes, 2015: a patientcentered approach: update to a position statement of the American Diabetes Association and the European Association for the Study of Diabetes. Diabetes Care. 2015; 38(1): 140-149. DOI: 10.2337/ dc14-2441.

2. Payk SL, Drew RH, Smith JD, Jiroutek MR, Holland MA. Sulfonylurea Prescribing Patterns After the Introduction of DPP-4 Inhibitors and GLP-1 Receptor Agonists. Clin Ther. 2015; 37(7): 1477-1482. DOI: http://dx.doi.org/10.1016/j. clinthera.2015.04.011.

3. Kalra S, Aamir AH, Raza A, Das A, Khan AA, Shrestha D, et al. Place of sulfonylureas in the management of type 2 diabetes mellitus in South Asia: a consensus statement. Indian J Endocrinol Metab. 2015; 19(5): 577-596. DOI: 10.4103/22308210.163171 .

4. Sharma M, Nazareth I, Petersen I. Trends in incidence, prevalence and prescribing in type 2 diabetes mellitus between 2000 and 2013 in primary care: a retrospective cohort study. BMJ Open. 2016; 6(1): 1-12. DOI: 10.1136/bmjopen-2015-010210.

5. Hampp C, Borders-Hemphill V, Moeny DG, Wysowski DK. Use of antidiabetic drugs in the US, 2003-2012. Diabetes Care. 2014; 37(5): 1367-1374. DOI: $10.2337 / \mathrm{dc} 13-2289$.

6. Fu H, Curtis BH, Schuster DP, Festa A, Kendall DM. Treatment patterns among older patients with type 2 diabetes in the United States: a Retrospective cohort study. Diabetes Technol Ther. 2014; 16(12): 833-839. DOI: 10.1089/dia.2014.0039.

7. Quaglia NB, Nuñez MH, Marzi MM. Drug prescriptions and demographic indicators in a diabetic population in Argentina. Farm Hosp. 2012; 36(2): 92-96. DOI: 10.1016/j.farma.2011.02.008.

8. Machado JE, Moncada-Escobar JC, Gaviria H. Quality and effectiveness of diabetes care for a group of patients in Colombia. Rev Panam Salud Publica. 2009; 26(6): 529-535. DOI: 10.1590/ S1020-49892009001200008.

9. Machado JE, Moncada JC, Mesa G. Antidiabetic drugs prescription patterns among a group of patients in Colombia. Rev Panam Salud Publica. 2007; 22(2): 124-131. DOI: 10.1590/S102049892007000700007.

10. Ministerio de Salud y Protección social de Colombia. Resolución Número 5592 de 2015, (2015).

11. Gimeno J, Lou L, Molinero E, Campos B, Boned B,
Sánchez Vañó R. Concordancia entre las fórmulas de Cockcroft Gault y del estudio MDRD para estimar la tasa de filtración glomerular en pacientes con diabetes tipo 2. Nefrología. 2006; 26(5): 615618.

12. Bolen S, Feldman L, Vassy J, Wilson L, Yeh $\mathrm{H}-\mathrm{C}$, Marinopoulos S, et al. Systematic review: comparative effectiveness and safety of oral medications for type 2 diabetes mellitus. Ann Intern Med. 2007; 147(6): 386-399. DOI: 0.7326/00034819-147-6-200709180-00178.

13. Chahal H. Comparative Safety and Efficacy of Glibenclamide in the elderly. Should elderly patients with type 2 diabetes be treated with glibenclamide (glyburide) or different sulfonylurea? Geneva, Switzerland: WHO, 2013.

14. Foundation NK. KDOQI clinical practice guideline for diabetes and CKD: 2012 update. Am J Kidney Dis. 2012; 60(5): 850-886.

15. Gómez R, Martínez A, Artola S, Górriz J, Menéndez E. Documento de Consenso sobre el tratamiento de la diabetes tipo 2 en el paciente con enfermedad renal crónica. Nefrología. 2014; 34(1): 34-45.

16. Kalra S, Gupta Y. Sulfonylureas. J Pak Med Assoc. 2015; 65(1):101-104.

17. Aires P, Gomes HV, Campos M. Management of hyperglycemia in patients with chronic kidney disease. J Nephrol. 2013; 26(4): 629-635. DOI: 10.5301/jn.5000248.

18. Al Khaja KA, Sequeira RP, Damanhori AH. Medication prescribing errors pertaining to cardiovascular/antidiabetic medications: a prescription audit in primary care. Fundam Clin Pharmacol. 2012; 26(3): 410-7. DOI: 10.1111/j.1472-8206.2011.00924.x.

19. American Geriatrics Society 2015 Beers Criteria Update Expert Panel. American Geriatrics Society 2015 updated beers criteria for potentially inappropriate medication use in older adults. J Am Geriatr Soc. 2015; 63(11): 2227-2246. DOI: 10.1111/jgs. 13702.

20. World Health Organization. 19th WHO Model List of Essential Medicines. 2015.

21. Sola D, Rossi L, Schianca GPC, Maffioli P, Bigliocca M, Mella R, et al. Sulfonylureas and their use in clinical practice. Arch Med Sci. 2015; 11(4): 840-8. DOI: 0.5114/aoms.2015.53304

22. Martínez A, Gorriz J, Segura J, Cebollada J, Escalada J, Esmatjes E, et al. Consensus document for the detection and management of chronic kidney disease. Nefrología. 2014; 34(2): 243-262. DOI: 10.3265 .

23. Guidoni CM, Borges A, Freitas O, Pereira 
LR. Prescription patterns for diabetes mellitus and therapeutic implications: a populationbased analysis. Arq Bras Endocrinol Metabol. 2012; 56(2): 120-127. DOI: 10.1590/S000427302012000200005.

24. Melander A, Lebovitz HE, Faber OK. Sulfonylureas Why, Which, and How? Diabetes Care. 1990; 13(Supplement 3): 18-25. DOI: 10.2337/ diacare.13.3.18.

25. Durán L, Salinas G, Zubieta A, Zendejas R, Zendejas J. Factores organizativos y profesionales asociados con la prescripción correcta de glibenclamida para el manejo de la diabetes mellitus tipo 2. Avances en Diabetología. 2012; 28(4): 95-101. DOI: 10.1016/j. avdiab.2012.09.001.

26. Zavala MA, Posada SE, Barrera L, López C, Mirón MT, Santiago P. Calidad de prescripción de hipoglucemiantes orales en una unidad médica familiar. Centro, Tabasco, México, 2009. Rev Mex Cienc Farm. 2011; 42(3): 42-49.

27. Zavala MA, Lima R, Gallegos MM. Utilización de hipoglucemiantes orales en una unidad médica familiar de Comalcalco, Tabasco, México, 2013. Rev Mex Cienc Farm. 2014; 45(3): 81-85.

28. Kalra S, Gupta Y, Saboo B. Essential drugs in diabetes: South and South East Asian perspective. J Soc Health Diabetes. 2015; 3(1): 4. DOI: 10.4103/2321-0656.140872.

29. Gangji AS, Cukierman T, Gerstein HC, Goldsmith $\mathrm{CH}$, Clase CM. A Systematic Review and MetaAnalysis of Hypoglycemia and Cardiovascular Events A comparison of glyburide with other secretagogues and with insulin. Diabetes Care. 2007; 30(2): 389-394. DOI: 10.2337/dc06-1789.

30. Simpson SH, Lee J, Choi S, Vandermeer B, Abdelmoneim AS, Featherstone TR. Mortality risk among sulfonylureas: a systematic review and network meta-analysis. Lancet Diabetes \& Endocrinol. 2015; 3(1):43-51. DOI: 10.1016/ S2213-8587(14)70213-X.

31. Torre EM, Tejedor FJL, Menéndez SA, NúñezCortés JM, García AA, Domingo MP, et al. Recomendaciones para el tratamiento farmacológico de la hiperglucemia en la diabetes tipo 2: Documento de consenso. Nefrología. 2011; 31(1): 17-26. DOI: 10.1016/j.aprim.2010.07.012.

32. Forst T, Hanefeld M, Jacob S, Moeser G, Schwenk G, Pfützner A, et al. Association of sulphonylurea treatment with all-cause and cardiovascular mortality: a systematic review and meta-analysis of observational studies. Diab Vasc Dis Res. 2013; 10(4): 302-314. DOI: 0.1177/1479164112465442. 